\title{
Former Students' Perception of Improvement Potential of Conceptual Modeling in Practice
}

\author{
Albert Tort, Antoni Olivé, and Joan A. Pastor \\ Department of Service and Information System Engineering \\ Universitat Politècnica de Catalunya - BarcelonaTech \\ \{atort, olive,pastor\}@essi.upc.edu
}

\begin{abstract}
Several authors have pointed out a significant gap between Conceptual Modeling (CM) theory and practice. It is then natural that we try to find answers to questions such as: What is the nature of the gap? Which is the magnitude of the gap? Why does the gap exist? and What could be done to narrow the gap? In this paper, we try to answer those questions from the point of view of the former students of a Requirements Engineering and Conceptual Modeling course that have been involved in professional projects. We have surveyed over 70 former students to know how they perceive the degree to which a set of four conceptual modeling artifacts are created in practice, and how they perceive the improvement potential of the creation of those artifacts in practice. For each artifact, we asked a question on the use of the artifact, and one on the recommendation of use of the artifact. We believe that there may be an improvement opportunity of the CM practice when a significant number of respondents would have recommended the creation of an artifact in the cases in which it was not created. We try to identify the reasons why the artifacts were not created, and what would be needed to convince stakeholders and developers to create the artifact, when it is recommended to do it.
\end{abstract}

Keywords: Conceptual modeling practice, Survey

\section{Introduction}

Several authors have pointed out a concern among researchers about a significant gap between Requirements Engineering and Conceptual Modeling (RE/CM) theory and practice $[12 / 314$. By theory, we mean here the mainstream methodologies and recommended best practices. If we accept that the gap exists, it is then natural that we try to find answers to questions such as: Which is the nature of the gap? Which is the magnitude of the gap? Why does the gap exist? and What could be done to narrow the gap?

There have been a few attempts to address those questions both in the CM field and in the broader RE field, but more research is needed to arrive at satisfactory answers [5]. 6] presents a multiple case study with 60 companies that draws 7 key issues in RE/CM practice. In [1, a state-of-the-practice survey was 
conducted in 12 SMEs, and three key RE/CM development needs were proposed for the future. To determine the actual use of conceptual modeling by practitioners and the most popular techniques and tools, [7/8, undertook an empirical study in Australia. 9] presents a qualitative study on 16 companies using agile software development approaches. [10] presents the analysis of in-depth interviews with 26 experienced conceptual modelers, and a descriptive theory of such practice. A diagnostic study of very small software companies in Chile is presented in 4]. 3] presents a survey assessing RE/CM practice in 27 Malaysian software firms, where most practicing professionals are graduated from the local educational institutions. A similar survey for New Zealand is presented in [11, where results are analyzed and benchmarked with best practices and with previous studies from Australia and New Zealand. Finally, 12 reports the results of a survey of business analysts designed to investigate the eventual mismatch between RE/CM education, training and practice, with a total of 9 preventing factors, with their implications for improvement.

In this paper, we focus on Conceptual Modeling (CM) practice, and we try to answer those questions from the point of view of the former students of a $\mathrm{RE} / \mathrm{CM}$ course that are, or have been, involved in projects with a significant CM activity. To this end, we have surveyed over 70 former students to know how they perceive the degree to which a representative set of CM artifacts are created in practice, and how they perceive the improvement potential of the creation of those artifacts in practice. In the literature, there are reports of surveys on former students' perceptions of the impact of the education they received on their professional activities [1213. However, as far as we know, this is the first time in which students of an informatics engineering university program are surveyed on the practice of CM.

A comprehensive analysis of CM practice should take into account the types of projects, the activities performed, the methods, techniques and tools used, and the artifacts created. Such an analysis is beyond the scope of this paper. Instead, here we focus on the creation in practice of the set of (closely-related) conceptual modeling artifacts consisting of the use cases, glossaries, structural schemas (or class diagrams) and integrity constraints. The reasons why we chose this focus were that the mentioned artifacts are: (1) widely-recognized as necessary artifacts in one form or another in most conceptual modeling projects; (2) easily identifiable in practice; and (3) well-known by the students.

For each artifact, we asked two main questions: one on the use of the artifact, and one on the recommendation of use of the artifact. Questions on usage are typical in most surveys [14, and in our case the question aimed at knowing the degree to which the artifact is explicitly created in practice. As far as we know, questions on recommendation have not been asked in similar surveys, and in our case the question aimed at knowing whether or not the respondent would have recommended the creation of the artifact when it was not created. We believe that there may be an improvement opportunity of the CM practice when a significant number of respondents would have recommended the creation of an artifact which is well-known by them in the cases in which it was not created. 
The structure of the paper is as follows. Section 2 briefly describes the $\mathrm{RE} / \mathrm{CM}$ course taken by the students that later participated in the survey. Section 3 describes how we designed and conducted the survey. Section 4 presents the general results of the survey. Section 5 presents the results for each of the four artifacts and points out a few implications for CM practice and research. Section 6 summarizes the conclusions and points out future work.

\section{The RE/CM Course}

In order to appreciate the results of the study reported in this paper, in this section we briefly describe the RE/CM course taken by the former students that participated in the survey.

The course started in 2005 as an elective course of the speciality in Software and Information Systems of the five-year program of Informatics Engineering taught at the Barcelona School of Informatics of the Universitat Politècnica de Catalunya (UPC) - BarcelonaTech. Typically, students take the course during their fourth year in the program, after (among others) an introductory course to software engineering.

The course is taught using a variant of the PBL (Project-Based Learning) approach. The main activity of the course is the requirements specification of a software system, including its complete conceptual schema. At the beginning of the course, the teachers establish a vision within an existing context [15], which varies each course. The students -working in groups of 5-7 people- have to study the relevant methods, languages and techniques and apply them to the determination and specification of the requirements of a system that realizes the vision.

The groups submit their work in two main deliverables: (1) Requirements Specification and (2) Conceptual Schema. Students have available selected deliverables from previous editions of the same course, which can be used as (good) examples. The conceptual schema (written in UML/OCL) must be formally defined using the USE tool [16], and be validated by means of example instantiations. The course emphasizes the artifacts of $\mathrm{RE} / \mathrm{CM}$, rather than the process used to develop them.

\section{Survey Design and Conduct}

We created a web-based survey [17] consisting of seven parts. The first part included two questions aiming at characterizing the number of years of professional experience, and the number of projects with a significant RE/CM activity in which the participant has been involved. Each of the other six parts focused on a specific $\mathrm{RE} / \mathrm{CM}$ artifact. In this paper we focus only on the four artifacts more closely related to conceptual modeling, which are (alternative names within parentheses): Use cases (scenarios), Glossary, Structural Schema (UML class diagram, ER schema) and Integrity Constraints (UML invariants). 
The names of the artifacts in the survey were as indicated above, but it was made clear that in practice they may be called with different names. It was also made clear that the questions referred to explicit artifacts written in any language, including natural language, and at any level of formality, not necessarily the same as those learnt in the $\mathrm{RE} / \mathrm{CM}$ course mentioned in the previous section.

The respondents were asked to answer the questions using a five-point Likert scale, with the values: 1 (never), 2 (rarely), 3 (sometimes), 4 (often) and 5 (always). The structure of each of the four artifact parts was essentially the same, and consisted of four subparts. There was a fifth part on the evaluation of the $\mathrm{RE} / \mathrm{CM}$ education received, but this part will not be analyzed here. The first subpart consisted of only one question $\mathcal{U}$ on the frequency of use of the artifact:

$\mathcal{U}$ : "In general, in the projects in which you have participated, the artifact was created ...?"

If the answer of the participant to $\mathcal{U}$ was less than 4 , then he was asked to answer the set of questions of the other three subparts described below. The first was the influence of five causes on the absence of the artifact in the projects in which he participated. In general, the causes suggested were:

- The methodology used did not require the creation of the artifact.

- It was considered too difficult to create the artifact.

- Stakeholders considered the artifact unnecessary or its cost not justified.

- There was an implicit definition of the artifact.

- Lack of tools for creating the artifact.

There was also an open-ended question for collecting other causes.

The next subpart was a single question $\mathcal{R}$ on the recommendation of use:

$\mathcal{R}$ : "In the projects in which the artifact was not created, would you have recommended its creation, taking into account the situation and the resources available at that time?"

This was a crucial question of the survey, because its answer gives a clear indication about the potential increase of use of the artifact in practice.

The last subpart asked about what would be needed in order to effectively create the artifact in practice. The suggested means were:

- To know what the artifact is and how to define it.

- To be convinced that the artifact is needed for system development.

- Better tools for creating the artifact.

- To be convinced that the cost of creating the artifact is worthwhile.

There was also an open-ended question for collecting other responses.

We targeted the survey to past students of the indicated RE/CM course. The potential number of survey participants was 369 , but we were able to know the current email address of 182 people (49.3\%). We sent them an email invitation (and reminders) to visit the survey website. We collected survey responses during October-December 2012. The survey was implemented using the web-based SurveyMonkey tool. The survey was initially tested through personal interviews with two former students with wide experience as practitioners. 
The survey participants were asked whether they were willing to participate in a post-survey focus group. A few of the most-experienced respondents that were willing to participate were invited to a 90-minutes meeting aimed at validating the survey results and conclusions. The discussions helped us to clarify answers and to point out improvement suggestions for increasing the use of CM in practice.

\section{Survey Result and Discussion}

In this Section, we describe the general results of the survey. In subsection 4.1 we summarize the number of participants in the survey by the number of years since they took the course, and the number of projects with a significant RE activity in which the participant has been involved. Subsection 4.2 provides an assessment of the use of each artifact in their current practice, and subsection 4.3 provides an assessment of the improvement potential in practice of each artifact.

\subsection{Participant Characteristics}

We received 72 complete responses to our survey, which represents a response rate of $39.6 \%$. Table 1 shows the percentage of participants by the number of years since the course was taken, and the number of projects with a significant $\mathrm{RE} / \mathrm{CM}$ activity in which the participant has been involved. It can be seen that the $55 \%$ of the participants took the course five or more years ago, and that the $61 \%$ have participated in three or more relevant projects. These results indicate that a large fraction of the respondents have a considerable experience in $\mathrm{RE} / \mathrm{CM}$. We call most-experienced respondents to those that have participated in more than three projects.

The table also shows that $6,94 \%$ of the respondents have not participated in any project with a significant RE/CM activity. These responses have been ignored in the results reported in this paper.

Table 1. Participants by number of years and projects (\%)

\begin{tabular}{c|cccccc|}
\multicolumn{7}{c}{ Projects } \\
\cline { 2 - 6 } Years & 0 & 1 & 2 & 3 & $>3$ & \multicolumn{1}{c}{} \\
\hline$\leq 2$ & 1.39 & 2.78 & 5.56 & 0.00 & 1.39 & $\mathbf{1 1 . 1 1}$ \\
3 & 0.00 & 4.17 & 6.94 & 5.56 & 2.78 & $\mathbf{1 9 . 4 4}$ \\
4 & 1.39 & 0.00 & 0.00 & 4.17 & 8.33 & $\mathbf{1 3 . 8 9}$ \\
5 & 2.78 & 4.17 & 2.78 & 1.39 & 16.67 & $\mathbf{2 7 . 7 8}$ \\
$\geq 6$ & 1.39 & 2.78 & 2.78 & 2.78 & 18.06 & $\mathbf{2 7 . 7 8}$ \\
\hline & $\mathbf{6 . 9 4}$ & $\mathbf{1 3 . 8 9}$ & $\mathbf{1 8 . 0 6}$ & $\mathbf{1 3 . 8 9}$ & $\mathbf{4 7 . 2 2}$ & \multicolumn{1}{l}{}
\end{tabular}


Table 2. Current practice by artifact

\begin{tabular}{l|ccc|ccc|}
\multirow{2}{*}{} & \multicolumn{4}{|c|}{ All } & \multicolumn{3}{c|}{ Most-experienced } \\
\cline { 2 - 7 } & M & SD & Mdn & M & SD & Mdn \\
\hline Use Cases & 3.10 & 1.13 & 3 & 3.09 & 1.04 & 3 \\
Glossary & 2.62 & 1.24 & 2 & 2.29 & 0.99 & 2 \\
Structural Schema & 3.56 & 1.20 & 4 & 3.48 & 1.18 & 4 \\
Integrity Constraints & 2.64 & 1.30 & 2 & 2.64 & 1.30 & 3
\end{tabular}

\subsection{Current Practice}

The first objective of our work was to obtain an assessment of the use of each $\mathrm{CM}$ artifact in practice, as perceived by former students. The assessment can be obtained from the answers to the $\mathcal{U}$ question. We computed the answer average in the Likert scale for each artifact.

Table 2 gives the mean $(\mathrm{M})$, standard deviation (SD) and median (Mdn) for each artifact, for all respondents, and for the most-experienced. It can be observed that there is very little disagreement between the perceptions of all respondents and that of the most-experienced. The largest disagreement is in the glossary, but the difference between the averages in this artifact (2.62 and 2.29 respectively) is only 0.33 .

In order to obtain an assessment of the improvement potential in practice of each artifact, we classified the situations in the current practice into two groups:

- Current Low Practice $(\mathcal{C L P})$. These are the situations in which the artifact is never or rarely or sometimes used (Likert scale 1, 2 or 3).

- Current High Practice $(\mathcal{C H P})$. These are the situations in which the artifact is often or always used (Likert scale 4 or 5).

Formally, the $\mathcal{C} \mathcal{L P}$ and $\mathcal{C H} \mathcal{P}$ of artifact $\mathcal{A}$ are defined as follows:

$$
\begin{gathered}
\mathcal{C} \mathcal{L}(\mathcal{A})=\frac{\mathcal{U}_{1}(\mathcal{A})+\mathcal{U}_{2}(\mathcal{A})+\mathcal{U}_{3}(\mathcal{A})}{\mathcal{U}(\mathcal{A})} * 100 \\
\mathcal{C H} \mathcal{P}(\mathcal{A})=\frac{\mathcal{U}_{4}(\mathcal{A})+\mathcal{U}_{5}(\mathcal{A})}{\mathcal{U}(\mathcal{A})} * 100
\end{gathered}
$$

where $\mathcal{U}_{i}(\mathcal{A}), i=1 . .5$, is the number of respondents that answered $i$ in the Likert-scale of the $\mathcal{U}$ question of artifact $\mathcal{A}$, and $\mathcal{U}(\mathcal{A})$ is the total number of respondents to that question.

Our rationale for the classification is that we consider unsatisfactory the $\mathcal{C} \mathcal{P}$ situations because the artifacts are created less than is expected by the theory, while the $\mathcal{C H} \mathcal{P}$ situations can be considered satisfactory because the artifacts are created at least often. Strictly speaking, we could consider unsatisfactory all situations in which the artifact is not created always, but we thought that, at least initially, we should accept as satisfactory the situations in which the 




Fig. 1. Current practice and improvement potential of each artifact

artifacts are created often or always. It would not be difficult to define $\mathcal{C} \mathcal{L P}$ and $\mathcal{C H P}$ in a more sophisticated way, taking into account the number of projects and the average value of the answers to the questions. However, we thought that our simpler and easier to understand definitions would suffice for our purpose.

Figure 1 shows two bars per artifact. The top bar corresponds to all respondents, while the bottom bar corresponds to the most-experienced respondents. Each bar has three segments. The left segment represents the value of $\mathcal{C H} \mathcal{P}$, and the rest of the bar (shown by two segments as will be explained later) represents the value of $\mathcal{C} \mathcal{L} \mathcal{P}$.

The artifact with the greatest value of $\mathcal{C H P}$ is the structural schema. The value is similar for both groups of respondents (close to 60\%). The artifacts with the least values of $\mathcal{C H P}$ are the glossary and the integrity constraints definition (25\%). However, for the most-experienced respondents, the $\mathcal{C H P}$ of glossaries is only the $17 \%$, which is very low.

The results shown in figure 1 provide a partial answer to the questions of What is the nature of the gap between CM theory and practice? and Which is the magnitude of that gap?:

- An aspect of the nature of the gap is that important CM artifacts are not created in practice as specified by the theory. According to that theory, the artifacts defining the use cases, glossary, structural schema and integrity constraints should be mandatorily created in most, if not all, CM projects, but they are not created in a significant number of them.

- The magnitude of the gap depends on the artifact. The smallest gap is in the structural schema (about 40\%). The largest is in the glossary (about 80\%). For the other artifacts, the gap lies between these two extremes. 


\subsection{Improvement Potential}

The second objective of our work was to obtain an assessment of the improvement potential in practice of each artifact, as perceived by former students. To this end, we asked to the former students in the $\mathcal{C} \mathcal{L}$ situation the $\mathcal{R}$ question, that we reproduce here:

$\mathcal{R}$ : "In the projects in which the artifact was not created, would you have recommended its creation, taking into account the situation and the resources available at that time?"

Based on the answer to this question, we say that there is:

- A situation with an Improvement Potential $(\mathcal{I P})$ if the answer was often (4) or always (5), and

- A situation of Accepted Low Practice $(\mathcal{A L P})$ if the answer was never (1), rarely (2) or sometimes (3).

Formally:

$$
\begin{gathered}
\mathcal{I P}(\mathcal{A})=\frac{\mathcal{R}_{4}(\mathcal{A})+\mathcal{R}_{5}(\mathcal{A})}{\mathcal{U}(\mathcal{A})} * 100 \\
\mathcal{A L P}(\mathcal{A})=\frac{\mathcal{R}_{1}(\mathcal{A})+\mathcal{R}_{2}(\mathcal{A})+\mathcal{R}_{3}(\mathcal{A})}{\mathcal{U}(\mathcal{A})} * 100
\end{gathered}
$$

where $\mathcal{R}_{i}(\mathcal{A}), i=1 . .5$, is the number of respondents that answered $i$ in the Likert-scale of the $\mathcal{R}$ question of artifact $\mathcal{A}$. Note that $\mathcal{C} \mathcal{L P}(\mathcal{A})=\mathcal{I P}(\mathcal{A})+$ $\mathcal{A L P}(\mathcal{A})$.

Our rationale for the definition of $\mathcal{I P}(\mathcal{A})$ is that we consider that situations have potential for improvement if they are in $\mathcal{C L P}$ but the respondents would have recommended often or always the creation of the corresponding artifact. That is, if the situation had followed the recommendation, then it would have been in the $\mathcal{C H} \mathcal{P}$ situation.

Similarly, our rationale for the definition of $\mathcal{A L P}$ is that we consider that situations remain in an unsatisfactory state if they are in $\mathcal{C} \mathcal{L}$ and the respondents would have not recommended often or always the creation of the corresponding artifact. That is, if the situation had followed the recommendation, then it would have remained in the $\mathcal{C L P}$ situation.

In Figure 1 the middle segment of each bar shows the value of $\mathcal{I P}(\mathcal{A})$, and the right segment shows the value of $\mathcal{A L P}(\mathcal{A})$.

The improvement potential of the four artifacts (in descending order) is: integrity constraints $(41 \%)$, use cases $(35 \%)$, structural schema $(27 \%)$, and glossary $(23 \%)$. The results can be considered similar for both groups of respondents.

These results indicate that our former students perceive a large room for improvement of the current situation in each artifact, specially in Integrity constraints and Use cases. The improvement potential of structural schemas and glossaries is similar, and lower, but their $\mathcal{C H} \mathcal{P}$ is quite different. 
Table 3. Reasons why the artifact was not created (average of answers)

\begin{tabular}{|c|c|c|c|c|c|c|c|c|}
\hline & \multicolumn{2}{|c|}{ Use Cases } & \multicolumn{2}{|c|}{ Glossary } & \multicolumn{2}{|c|}{ Struct. Schema } & \multicolumn{2}{|r|}{ ICs } \\
\hline & All & Most-exp & All & Most-exp & All & Most-exp & All & Most-exp \\
\hline $\begin{array}{l}\text { Methodology did not } \\
\text { require it }\end{array}$ & 2.63 & 2.77 & 2.62 & 2.55 & 2.92 & 3.27 & 2.39 & 2.43 \\
\hline Too difficult to create it & 2.80 & 2.23 & 2.04 & 2.03 & 2.75 & 2.91 & 2.95 & 3.13 \\
\hline $\begin{array}{l}\text { Stakeholders consid- } \\
\text { ered it unnecessary or } \\
\text { its cost not justified }\end{array}$ & 3.20 & 3.18 & 3.74 & 3.69 & 3.33 & 3.36 & 3.55 & 3.57 \\
\hline $\begin{array}{l}\text { There was an implicit } \\
\text { definition }\end{array}$ & 3.45 & 3.59 & 3.72 & 3.72 & 2.92 & 2.55 & 3.16 & 2.78 \\
\hline Lack of tools & 2.55 & 2.41 & 1.98 & 1.76 & 2.58 & 2.45 & 2.66 & 2.52 \\
\hline
\end{tabular}

\section{Results and Discussion per Artifact}

In this section we focus on each of the four artifacts. We explain the main results of the survey for the artifact, and their implications for practice and research in CM. The four artifacts were handled in a uniform way, and the answers are summarized in Tables 3 and 4.

\subsection{Use Cases}

As illustrated in Fig. 1, $\mathcal{C H P}$ (use cases) $=38 \%$ (35\% if we only take into account the most-experienced respondents). This means that over $60 \%$ of the respondents perceive that use cases are defined either never or rarely or sometimes. To these respondents, the survey asked the reasons why the artifact was not created. The answers averages are shown in Table 3.

The participants in the validation meeting agreed that often use cases are not valuable deliverables for customers, who expect "working" artifacts as soon as possible.

The survey also asked what would be needed for convincing stakeholders and developers that it is necessary and useful to create the artifact. The results are shown in 4

Implications for Practice and Research: According to the former students, the improvement potential of use cases is $35 \%$ ( $27 \%$ for the most-experienced). This means that the current high practice could be almost doubled.

In order to achieve that improvement in practice, it is necessary, first of all, to increase the knowledge of the artifact among practitioners. This seems feasible using an adequate professional training program. Once use cases are known, it should not be difficult to convince practitioners that:

- use cases are always created, although they often remain in the minds of developers (implicit), 
Table 4. What would be needed for convincing stakeholders that is necessary and useful to create the artifact (average of answers)

\begin{tabular}{|c|c|c|c|c|c|c|c|c|}
\hline & \multicolumn{2}{|c|}{ Use Cases } & \multicolumn{2}{|c|}{ Glossary } & \multicolumn{2}{|c|}{ Struct. Schema } & \multicolumn{2}{|r|}{ ICs } \\
\hline & All & Most-exp & All & Most-exp & All & Most-exp & All & Most-exp \\
\hline $\begin{array}{l}\text { To know the artifact } \\
\text { and how to define it }\end{array}$ & 3.55 & 3.45 & 2.98 & 3.10 & 3.71 & 3.82 & 3.73 & 3.78 \\
\hline $\begin{array}{l}\text { To be convinced that } \\
\text { the artifact is needed }\end{array}$ & 4.03 & 3.95 & 3.83 & 3.90 & 4.33 & 4.36 & 4.00 & 3.96 \\
\hline Better tools & 3.23 & 3.00 & 2.40 & 2.28 & 3.21 & 3.00 & 2.95 & 2.61 \\
\hline $\begin{array}{l}\text { To be convinced that } \\
\text { the cost of creating the } \\
\text { artifact is worthwhile }\end{array}$ & 4.10 & 4.00 & 3.83 & 3.86 & 4.13 & 4.27 & 3.74 & 3.55 \\
\hline
\end{tabular}

- therefore, the cost of writing use cases (making them explicit) is very low, and

- use cases are useful not only for system specification, but also for development planning, testing and documentation.

An implication for research is the recognized need for better tools. There are already in the market several tools that help in the creation of use cases, but the challenge is to make those tools (or others) appropriate for any type of project.

\subsection{Glossary}

As illustrated in Fig. 1. $\mathcal{C H} \mathcal{P}$ (glossary) $=25 \%$ (12\% if we only take into account the most-experienced respondents). These data indicate that glossaries are perceived as the least used artifacts. On the other hand, it is noticeable that the most experienced responders perceive a much lower level of high practice of glossaries.

This means that over $75 \%$ of the respondents perceive that glossaries are defined either never or rarely or sometimes. To these respondents, the survey asked the reasons why the artifact was not created. The results are shown in Table 3. It is noticeable that glossaries are the artifacts for which the reason "implicit definition" has the greatest average value (3.72), and the reason "lack of tools" has the lowest one (1.98).

In the open-ended question, several respondents insisted on the fact that glossaries were not needed in their projects. The participants in the validation meeting explained that the terminology used in each project/domain is learned by practice during discussions with the stakeholders.

The survey also asked what would be needed for convincing stakeholders and developers that it is necessary and useful to create the artifact. The answers averages are shown in Table 4 . 
The three respondents to the open-ended question indicated that glossaries are not needed when the meaning of the terms is already known by the involved people.

Implications for Practice and Research: According to the former students, the improvement potential of the glossaries is $23 \%$ ( $17 \%$ for the most-experienced). This means that the current high practice could be increased by about $100 \%$. However, even if the improvement potential were achieved in full, the resulting high practice would remain below $50 \%$.

To our view, the results of the survey suggest that the theory concerning glossaries does not adequately deal with project settings in which project participants think that they already agree on the meaning of terms and, therefore, that they do not need defining those terms in glossaries.

\subsection{Structural Schema}

As illustrated in Fig. 1. $\mathcal{C H} \mathcal{P}$ (structural schema) $=59 \%$ (64\% if we only take into account the most-experienced responders). These data indicate that structural schemas are perceived as the most used artifacts in CM practice. This result is consistent with that of [18, which found that UML class diagrams were the most frequently used of seven UML components (including use case diagram and narrative).

About $40 \%$ of the respondents perceive that structural schemas are defined either never or rarely or sometimes. To these respondents, the survey asked the reasons why the artifact was not created. The results are shown in Table 3 .

Our results differ from those of 18 . A logical difference is that they found as main reason for UML diagrams not being used "a lack of understanding by analysts", differing from our respondents who are trained in CM artifacts. Another difference is that our respondents give higher importance to the reason "insufficient value to justify the cost".

According to the former students, the improvement potential of structural schemas is $27 \%$ ( $21 \%$ for the most-experienced). This means that the current high practice could be increased by about $50 \%$. Therefore, structural schemas are the artifacts with the lowest relative potential increase with respect to the current high practice, although that practice is already the largest one.

The survey also asked what would be needed for convincing stakeholders and developers that it is necessary and useful to create the structural schema. The answers averages are shown in Table 4 .

Some participants in the validation meeting explained that the level of formalism of the conceptual schema depends on the methodology (e.g. when agile practices are applied only an iterative sketch is defined) but they confirmed that some kind of conceptual schema specification is widely used for internal development purposes. Nevertheless, they suggested that better generation of prototypes and executable models from the conceptual schema could be very useful in order to make the conceptual schema a valuable artifact from the customer point of view. 
Implications for Practice and Research: In order to achieve the potential improvement in practice, it is necessary, first of all, to increase the knowledge of the structural schemas among practitioners. This seems feasible using an adequate professional training program. Once structural schemas are known, it should not be difficult to convince practitioners that:

- structural schemas are always created, independently from the methodology used, although they often remain in the minds of developers (implicit),

- therefore, the cost of writing structural schemas (making them explicit, more or less formally) should be low for trained practitioners, and

- structural schemas, due to their graphical representation, are recognized as one of the best means for improving the communication between the parties involved in the development process.

An implication for research is the recognized need for better tools. There are already in the market several tools that help in the creation of structural schemas, but the challenge is to make those tools (or others) convenient for use in any type of project where structural schemas are (or need to be) created.

\subsection{Integrity Constraints}

As illustrated in Fig. 1. $\mathcal{C H} \mathcal{P}$ (integrity constraints) $=27 \%$. Exactly the same value is obtained if we only take into account the most-experienced responders. These data indicate that the definition of integrity constraints is perceived as one of the least created artifacts. The survey asked the reasons why integrity constraints were not defined. The answers averages are shown in Table 3 .

Both groups of respondents considered that the artifact of integrity constraints is the most difficult to create. In the validation meeting, the participants suggested that integrity constraints are not usually considered necessary because they are implicitly assumed. However, they explained several experiences in which an early identification of constraints would have improved the result and reduced the cost of the project.

According to the former students, the improvement potential of integrity constraints definition is $41 \%$ ( $46 \%$ for the most-experienced). This means that the current high practice could increase by over $150 \%$. Therefore, integrity constraints definition is one of the two artifacts with the largest relative potential increase with respect to the current high practice.

The survey also asked what would be needed for convincing stakeholders and developers that it is necessary and useful to explicitly define the integrity constraints. The answers averages are shown in Table 4.

Implications for Practice and Research: In order to achieve the improvement in practice, it is necessary to increase the knowledge of the artifact among practitioners, for example by using an adequate professional training program. Once integrity constraints are known, it should not be difficult to convince practitioners that:

- integrity constraints are necessary to ensure the integrity of the system,

- lack of integrity normally has negative consequences. 


\section{Conclusions}

In this paper, we have focused on the recognized gap between CM theory and practice, and we have addressed the questions of: What is the nature of the gap? Which is the magnitude of the gap? Why does the gap exist? and What could be done to narrow the gap? To find (at least partial) answers to those questions in our local context, we have surveyed over 70 former university students to know how they perceive the degree to which a set of four CM artifacts are created in practice, and how they perceive the improvement potential of the creation of those artifacts in practice. The artifacts were the use cases, glossary, structural schema and integrity constraints.

We have shown that (one aspect of) the nature of the gap is that important $\mathrm{CM}$ artifacts are not created in practice as specified by the theory. According to that theory, the above mentioned artifacts should be mandatorily created in most, if not all, CM projects, but we have seen that they are not created in a significant number of cases.

We have shown that the magnitude of the gap depends on the artifact. The smallest gap is in the structural schema, and it is about $40 \%$. The largest is in the glossary, and it is about $80 \%$. For the other artifacts, the gap lies between these two extremes.

We have described the reasons why the gap exists. We have seen that the reasons depend on the artifact considered. In general, the two main reasons are that in practice there is an implicit definition of the artifact, and that stakeholders consider the explicit artifact unnecessary, or its cost not justified.

We have shown that the improvement potential of the four artifacts (in descending order) is: Integrity constraints (41\%), Use cases (35\%), Structural schema $(27 \%)$, and Glossary (23\%). These results indicate that the former students perceive a large room for improvement of the current situation.

We have suggested ideas on what should be done to achieve the improvement potential. This depends also on the artifact. In general, what is needed is to convince the stakeholders that the creation of the artifact is needed, and that the cost of creating it is worthwhile. A few implications for practice and research that may help achieving the improvement have been suggested.

As is usual in similar research works [12, the results reported in this paper are subject to some threats to their validity beyond our local context, which we can only summarize here. One is the geographic and domain bias created by drawing the respondents from the former students of an $\mathrm{RE} / \mathrm{CM}$ course offered by a particular university. Another possible threat is bias introduced by the form of the questions asked in the questionnaire. The closed set of responses might have led respondents to available responses rather than take more time to provide open-ended answers. However, the validation meeting we held allowed us to validate and, in some cases, to clarify the results of the survey.

The work reported here can be extended in several directions. Here, we just suggest: (1) taking into account the type and size of the projects and of the companies in which the participants have worked, and (2) analyzing the execution in practice of critical CM activities. 


\section{References}

1. Nikula, U., Sajaniemi, J., Kälviäinen, H.: A State-of-the-practice Survey on Requirements Engineering in Small-and Medium-sized Enterprises. Lappeenranta University of Technology (2000)

2. Neill, C.J., Laplante, P.A.: Requirements engineering: The state of the practice. IEEE Software 20(6) (2003) 40-45

3. Tahir, A., Ahmad, R.: Requirement engineering practices-an empirical study. In: CiSE 2010, IEEE (2010) 1-5

4. Quispe, A., Marques, M., Silvestre, L., Ochoa, S.F., Robbes, R.: Requirements engineering practices in very small software enterprises: A diagnostic study. In: SCCC 2010, IEEE (2010) 81-87

5. Anaby-Tavor, A., Amid, D., Fisher, A., Ossher, H., Bellamy, R., Callery, M., Desmond, M., Krasikov, S., Roth, T., Simmonds, I.: An empirical study of enterprise conceptual modeling. In: ER 2009, Springer (2009) 55-69

6. Emam, K.E., Madhavji, N.H.: A field study of requirements engineering practices in information systems development. In: 2nd IEEE International Symposium on Requirements Engineering, IEEE (1995) 68-80

7. Davies, I., Green, P., Rosemann, M., Gallo, S.: Conceptual modelling-what and why in current practice. In: ER 2004, Springer (2004) 30-42

8. Davies, I., Green, P., Rosemann, M., Indulska, M., Gallo, S.: How do practitioners use conceptual modeling in practice? Data and Knowledge Engineering 58(3) (2006) 358-380

9. Cao, L., Ramesh, B.: Agile requirements engineering practices: An empirical study. Software, IEEE 25(1) (2008) 60-67

10. Milton, S.K., Rajapakse, J., Weber, R.: Conceptual modeling in practice: An evidence-based process-oriented theory. In: ICIAFs 2010, IEEE (2010) 533-536

11. Talbot, A., Connor, A.: Requirements engineering current practice and capability in small and medium software development enterprises in new zealand. In: SERA 2011, IEEE (2011) 17-25

12. Wever, A., Maiden, N.: What are the day-to-day factors that are preventing business analysts from effective business analysis? In: RE 2011, IEEE (2011) 293-298

13. University of Idaho: 2006 survey of graduates classes of 1998, 1999, 2000 and 2001. Technical report (2007) Available at http://www.webs.uidaho.edu/ira/assess/ grad_alum_survey/GAS\%20UI\%20Summary $\% 202011-2012 \% 20$.pdf

14. Hitchman, S.: Object-oriented modelling in practice: class model perceptions in the ERM context. In: ER 2000, Springer (2000) 397-408

15. Jarke, M., Pohl, K.: Establishing visions in context: Towards a model of requirements processes. In: ICIS 1993. (1993) 23-24

16. Gogolla, M., Büttner, F., Richters, M.: Use: A UML-based specification environment for validating UML and OCL. Science of Computer Programming 69(1-3) (2007) 27-34

17. Tort, A., Olivé, A., Pastor, J.A.: Survey on requirements engineering and conceptual modeling in practice. Technical report, Universitat Politècnica de Catalunya (2013) Available at http://www.essi.upc.edu/ gmc/SurveyREPractice2012.pdf

18. Dobing, B., Parsons, J.: How UML is used. Communications of the ACM 49(5) (2006) 109-113 